\title{
The main reasons of rebound, coagulation, and explosive disintegration of the liquid drops in gas-vapor-droplet streams
}

\author{
Ivan Voytkov ${ }^{1}$, Olga Vysokomornaya $^{1, *}$, Maksim Piskunov $^{1}$, and Nikita Shlegel ${ }^{1}$ \\ ${ }^{1}$ National Research Tomsk Polytechnic University, 634050 Tomsk, Russia
}

\begin{abstract}
We have reviewed the known data about mechanisms, conditions, reasons, and characteristics of rebound, coagulation, and explosive disintegration of drops in gas-vapor-droplet streams. The three main factors of altering a direction of motion, a velocity, a size and a concentration of droplets in a gaseous stream have been highlighted. Among of them are the thermal factor (heating and evaporation of drops), the aerodynamic factor (acceleration, reverse motion, and deceleration of drops due to their entrainment by gaseous stream), the dynamic factor (change in size, velocity, and direction of motion of drops after their collision). We have generalized the findings obtained by various authors in experiments with two drops, little group of drops, and aerosol. In addition, we have reviewed the published results about the fields of velocities and motion trajectories of drops in a flow, about the change in size and concentration of drops due to rebound, coagulation, disintegration (i.e. the complete destruction of the parent drops and the detachment of the liquid fragments of different size and volume from the surface).
\end{abstract}

\section{Introduction}

Gas-vapor-droplet technologies present a wide group of practical applications based on the interaction between the flows of sprayed liquid and gas. A considerable part of such technologies includes heating or evaporation of a liquid in the medium of hot gases. Among such applications are the contact heat exchangers [1], the drier installations [2], the technologies of the polydisperse fire suppression [3], the waste-heat recovery by a formation of gas-vapor heat carriers [4], the thermal purification of wastewater [5], etc. In addition to the mentioned heat and power technologies, as well as firefighting solutions, it is extremely important to consider structures and compositions of gas-vapor-droplet flows when treating of different surfaces by atomization (protective and paint coatings), in the fuel-feed systems, in the meteorology, medicine [6,7], etc.

Currently, the three main groups of factors of altering a direction of motion, a velocity, a size and a concentration of droplets in a gaseous stream are known. Among them are the thermal factor (heating and evaporation of drops) [8-10], the aerodynamic factor

\footnotetext{
* Corresponding author: vysokomornaja@tpu.ru
} 
(acceleration, reverse motion, and deceleration of drops due to their entrainment by gaseous stream) $[8,11]$, the dynamic factor (change in size, velocity, and direction of motion of drops after their collision) [12-17].

The purpose of the study is to review the state-of-art concepts about the three main mechanisms of varying the drop concentration in the gas-vapor-droplet flows.

\section{Thermal Factor}

As known, liquid evaporation occurs even at relatively low temperatures. However, with an increase in temperature, an evaporation rate nonlinearly grows. Homogeneous drops usually evaporate monotonously from a free surface. This process is accompanied by a step-down of their size [18]. Numerous studies focus on an analysis of particularities and characteristics of the liquid evaporation in a dropwise state. However, to date, an adequate prediction of the mass rate of evaporation is possible in a limited range of temperature only, up to $600 \mathrm{~K}$. A more tremendous challenge is to describe a behavior of the evaporating heterogeneous drops because a presence of the interfaces causes the inhomogeneous fields of temperatures and concentrations, the vortices, and even the vapor bubbles that can result in disintegrating a drop.

A controlled use of a disintegration of the heterogeneous drops when heated can be applied in not only conventional heat and power technologies [1-5], but also in the presentday technologies of formation and combustion of the composite fuels based on wastes of the coal conversion and the oil refining [19].

The study of mechanisms, modes, and characteristics of heat and mass transfer in the case of the drop disintegration when heated [18] has enabled us to highlight the main governing factors. Among them are nucleate points (solids, bubbles of dissolved gases, etc.), differences in thermos-physical properties of components of the heterogeneous drops, a dominant heating mechanism, thermal radiation transmittance and accumulation, etc.

In addition, studies $[20,21]$ state that the intermolecular interactions and the influence of the drop components against each other on a change in their thermos-physical properties during heating can play a defining role. In particular, when heating the $0.1 \%$ solution of surfactant in water becomes less transparent in comparison with pure water. As a result, an aggregation of the amphiphilic molecules occurs. Based on experiments [20, 21], a correlation between temperatures of the water-based solutions and a level of turbidity has been found. An analysis of thermocouple measurements and experimental data obtained using highly precise microscopes confirms the conclusions drawn in studies [20, 21].

\section{Aerodynamic Factor}

One of the main problems of predicting a behavior of the gas-vapor-droplet streams is a difficulty to control an entrainment of drops by gases [22, 23]. Researches [8, 11] provide data about the main characteristics of phase transformations and deformation of drops of the polydisperse water flow under conditions close to the technologies with gas-vapordroplet media [1-7]. Studies [9, 24] generalize, in a dimensionless form, the experimental data presented in $[22,23]$. The results presented in such a manner characterize the conditions for deceleration and possible subsequent entrainment of drops by hightemperature gases.

An analysis of the experimental results presented in $[10,25]$ allows us to deduce that the drops of water and the water-based emulsions keep an integrity during motion through the gaseous medium in the conditions of varying their size and velocity in a limited range. It is assumed that water drops disintegrate in the gaseous flow when the Weber numbers are 
10-12 [25, 26]. Study [27] reports that during motion of water drops through hightemperature combustion products the limit Weber numbers are 9-11. In addition, research [27] considers an influence of velocity and size of drops on characteristics of their deformation and an influence of the temperatures of gaseous medium and drops on characteristics of these processes. Moreover, the authors of study [27] show an effect of the aerodynamic factor at a variation of velocity and size of drops. Consequently, the links between the temperature gradients at the liquid / gas interface and the characteristics of deformation of water drops moving through a gaseous medium have been found.

Research [28] reports that the limit Weber numbers for the drops of mercury, alcohol, and kerosene are about 10. Study [29] describes that for the drop moving through the gaseous flow the critical Reynolds number characterizing a transition from the laminar flow regime to the turbulent one is about $3 \cdot 10^{5}$.

\section{Drop Interaction Factor}

Another factor considerably influencing the gas-vapor-droplet stream characteristics is a constant collision of the liquid drops. Currently, in the published studies researchers have try to find regimes of the drops collision and probabilities of consequences corresponding to these regimes [12-17].

To consider such factors as size of the colliding drops, their thermos-physical properties and velocity, the dimensionless criterion (the Weber number (We) can be used. Studies [12-17] provides the following scenarios of collisions: (1) coagulation; (2) rebound; (3) short-term coagulation with subsequent disintegration and formation of the secondary drops, which are similar in size to the primary ones; (4) collision scenario when the drop passes through another one; (5) explosive disintegration of drops with a formation of great number of small sub-droplets. The Weber numbers ranges have been obtained based on experiments for each collision scenario.

Note that studies [12-17] use the similar experimental methodologies. However, the results on the Weber numbers ranges considerably differ. In particular, the scenarios of the explosive disintegration and the passing the drop through another one in [12] are characterized by $\mathrm{We}>25$ and $\mathrm{We}>50$, respectively. Whereas, in $[13,14]$ the scenario of passing the drop through another one occurs at $\mathrm{We}>50$, but the explosive disintegration scenario - at We $>100$. Moreover, the authors of researches [12-14] show zero probabilities of occurrence of any other consequences of collisions in each range mentioned. At the same time, studies [16, 17] depict the insignificant but not zero probability of occurrence of each scenario in any Weber numbers range.

The authors of studies [13-16] make several assumptions about the possible reasons for occurring each scenario of the drops collision consequences. In particular, the reason for coagulation of drops at small Weber numbers can be the drops surface oscillation of and the diminished pressure in the airspace between them. The rebound of drops stems from a presence of the gas space between them. The possible reason for the short-term coagulation with the subsequent disintegration is a rotational movement of drops due to a tangential collision. Lastly, the explosive disintegration of drops arises from cavitation at high kinetic energy during collisions.

\section{Current Issues}

The most challenging task of studies considering the thermal, aerodynamic, and dynamic factors is a regulation of the possible ranges of increase in surface area of liquid in the gaseous medium during the drop disintegration. To date, there is no comparative 
assessment describing a manifold increase in the surface area of liquid in the gaseous medium by using one of the factors considered in the article. Such an assessment is of particular importance for heat-exchange technologies to attain an enhancement of phase transitions [30, 31]. Most likely, the maximum efficiency can be reached when using a combined effect of thermal, aerodynamic, and dynamic factors, during the collisions of the heterogeneous drops heated up to high temperatures. These conditions are often fulfilled in multiphase and multi-component gas-vapor-droplet streams, but while they are underexplored. A detailed analysis of change in structure and composition of such streams is of importance for understanding their main parameters and the considerable resource saving in the appropriate technologies.

\section{Conclusion}

The analysis of literature devoted to the study of characteristics of the gas-vapor-droplet streams allows us to deduce that over a period of several decades results of such investigations remain in high demand. During this time, researchers have obtained numerous experimental and analytical findings enabling the predictions of the main parameters of the gas-vapor-droplet stream, their dynamics depending on many factors, namely, gas temperature, velocities of drops and a gaseous medium, droplet size distribution, etc. However, a difference in the results obtained by various authors is most likely due to an influence of the conditions, which have not been considered yet up to date in studies, namely, shapes of drops during collisions, properties of a gaseous medium, a presence of the vapor film around the evaporating drops, etc. The most complete consideration of all the particularities of fluid dynamics and heat mass transfer will considerably expand a theory of the formation of the gas-vapor-droplet streams and provide a basis for the development of the adequate methods for predicting their characteristics.

Research was supported by the Russian Science Foundation (project 18-71-10002).

\section{References}

1. R. M. Schirmer, R. L. Eson, J. Petrol. Technol. 37 (10), 1903 (1985)

2. M. G. Lee, Jajaei P. Mansouri, S. Mansouri, K. Hapgood, M. W. Woo, Dry. Technol. 36 (2), 178 (2018)

3. Z. Tang, Z. Fang, J. P.Yuan, B. Merci, Fire Saf. J. 55, 35 (2013)

4. M. Ren, S. Wang, L. Qian, Y. Li, Appl. Mech. Mater. 577, 523 (2014)

5. P. R. von Rohr, K. Prikopsky, T. Rothenfluh, Strojnicky Casopis 59 (2), 91 (2008)

6. D. Tarlet, C. Josset, J. Bellettre, Int. J. Heat Mass Transfer. 95, 689 (2016)

7. K. Sollohub, K. Cal, J. Pharmaceut. Sci. 99 (2), 587 (2010)

8. G. V. Kuznetsov, P. A. Strizhak, High Temp. 52 (4), 568 (2014)

9. R. S. Volkov, G. V. Kuznetsov, P. A. Strizhak, J. Eng. Phys. Thermophys. 88 (4), 937 (2015)

10. R. S. Volkov, O. V. Vysokomornaya, G. V. Kuznetsov, P. A. Strizhak, Adv. Mech. Eng. 2014, 865856 (2014)

11. G. V. Kuznetsov, P. A. Strizhak, Techn. Phys. Lett. 40 (6), 499 (2014)

12. D. G. Pazhi, V. S. Galustov, Basic techniques of spraying liquids [Osnovy techniki raspylivaniya zhidkostey] (Chimiya Publ., Moscow, 1984) [in Russian] 
13. V. A. Arkhipov, V. F. Trofimov, J. Appl. Mech. Tech. Phys. 46 (1), 42 (2005)

14. V. A. Arkhipov, G. S. Ratanov, V. F. Trofimov, J. Appl. Mech. Tech. Phys. 19 (2), 201 (1978)

15. V. A. Arkhipov, I. M. Vasenin, V. F. Trofimov, J. Appl. Mech. Tech. Phys. 24 (3), 371 (1983)

16. D. V. Antonov, R. S. Volkov, G. V. Kuznetsov, P. A. Strizhak, J. Eng. Phys. Thermophys. 89 (1), 100 (2016)

17. R. S. Volkov, G. V. Kuznetsov, P. A. Strizhak, Techn. Phys. Lett. 41 (9), 840 (2015)

18. O. V. Vysokomornaya, I. S. Voytkov, G. V. Kuznetsov, A. V. Abramova, Int. J. Heat Mass Transf. 126, 1043 (2018)

19. D. O. Glushkov, P. A. Strizhak, M. Yu. Chernetskii, Therm. Eng. 63 (10), 707 (2016)

20. A. V. Kim, N. N. Medvedev, A. Geiger, 2014. J. Mol. Liq. Spec. issue: Fluid phase associations 189, 74 (2014)

21. V. P. Voloshin, A. V. Kim, N. N. Medvedev, R. Winter, A. Geiger, Biophys. Chem. 192, 1 (2014)

22. R. S. Volkov, G. V. Kuznetsov, P.A. Strizhak, Int. J. Therm. Sci. 88, 193 (2015)

23. A. Maatar, S. Chikh, M. Ait Saada, Int. J. Heat Mass Transf. 86, 212 (2015)

24. Yu. V. Alekhanov, M. V. Bliznetsov, Yu. A. Vlasov, V. I. Dudin, A. E. Levushov, A. I. Logvinov, S. A. Lomtev, E. E. Meshkov, Techn. Phys. Lett. 29 (3), 218 (2003)

25. J. Eggers, E. Villermaux, Rep. Progr. Phys. 71, 036601 (2008)

26. J. E. Sprittles, Y. D. Shikhmurzaev, Phys. Fluids 24, 122105 (2012)

27. R. S. Volkov, A. O. Zhdanova, G. V. Kuznetsov, P. A. Strizhak, Techn. Phys. 60 (10), 1443 (2015)

28. V. V. Dubrovskiy, V. V. Podvysotskiy, A. A. Shrayber, Inzhenerno-fizicheskiy Zhurnal 58 (5), 804 (1990) [in Russian]

29. E. H. Trinh, R. G. Holt, D. B. Thiessen, Phys. Fluids 8 (1), 43 (1996)

30. O. V. Vysokomornaya, M. V. Piskunov, P. A. Strizhak, App. Therm. Eng. 127, 1340 (2017)

31. P. A. Strizhak, M. V. Piskunov, R. S. Volkov, J. C. Legros, Chem. Eng. Res. Des. 127, 72 (2017) 